\title{
Análisis de la evolución de las áreas ambientalmente sensibles a la desertificación en la cuenca del Puangue en Chile
}

\author{
Analysis evolution of environmentally sensitive areas to desertification in the \\ Puangue river basin Chile
}

Ana Isabel Huaico Malhue ${ }^{*}$

\begin{abstract}
RESUMEN
La desertificación es un proceso silencioso que afecta a la región mediterránea de Chile central. Este estudio analiza la evolución de la sensibilidad ambiental a la desertificación en la cuenca agrícola del estero Puangue en la Región Metropolitana de Santiago de Chile. Se utiliza el modelo desarrollado por el proyecto MEDALUS (Mediterranean Desertification and Land Use) en el área de estudio. Se concluye que la sensibilidad a la desertificación ha aumentado en los últimos 30 años debido principalmente al impacto del cambio de uso a agrícola en los sectores de montaña.
\end{abstract}

Palabras clave: agricultura, desertificación, áreas ambientalmente sensibles

\begin{abstract}
Desertification is a silent process that affects the mediterranean region in central Chile. This study analyzes the evolution of environmental sensitivity to desertification in the Puangue river basin in the Metropolitan Region of Santiago de Chile. The model developed by the MEDALUS project (Mediterranean Desertification and Land Use) is used in the study area. It is concluded that the sensitivity to desertification has increased in the last 30 years. The foregoing mainly due to the impact of the change of use to agriculture in the mountain sectors.
\end{abstract}

Key words: agricultural, desertification, environmentally sensitive areas

\section{Introducción}

La desertificación se define como la degradación de tierras en zonas áridas, semiáridas y subhúmedas secas resultante de diversos factores, entre ellos, las variaciones climáticas y las actividades humanas (UNEP, 1992). La población total asentada en estos ambientes en el mundo se estima en unos 2.000 millones (UNCCD, 2011) y con una proyección creciente, por lo que la demanda de recursos naturales en esas áreas va a ir en aumento. Esto, ante un escenario de cambio climático (IPCC, 2014), es preocupante.

El 58\% del territorio de Chile es árido, lo que corresponde al 6\% $\left(435.991 \mathrm{~km}^{2}\right)$ del total de las zonas áridas de América Latina (Verbist, 2010). La información sobre las zonas áridas es escasa y el monitoreo de la erosión, un factor clave de la desertificación, sobre todo en las escalas locales a nivel país, es mínimo (Pizarro et al., 2010).

Estudios realizados por la Universidad de Chile (1997) indican que la degradación de los recursos del área mediterránea árida y semiárida de Chile es un proceso continuo y sostenido que conduce a estados de deterioro cada vez más agudos. Más recientemente, el Congreso Nacional (2012) advirtió que la productividad agrícola en el país, un indicador de degradación, disminuyó su crecimiento de 2,8\% entre 1984-1997, a sólo 0,9\% entre 1998 y 2005. Estos mismos antecedentes señalan que el fenómeno

\footnotetext{
1 Municipalidad de María Pinto. María Pinto, Chile.

* Autor por correspondencia: ahuaico@mpinto.cl
} 
se expresa con mayor magnitud en las macrozonas agroecológicas de la precordillera de la I y II Región, la faja costera de la I a la IV Región, las áreas ocupadas por las comunidades agrícolas de la III a la IV Región, el secano costero de la V a la VIII Región, la precordillera andina de la VI a la VIII Región y las zonas degradadas de la XI a la XII Región.

De acuerdo con Gardi et al., (2014), la superficie chilena afectada por la desertificación asciende a 47,3 millones de hectáreas (aproximadamente el $62 \%$ del territorio nacional). Según estos autores, la erosión constituye en la actualidad uno de los problemas ambientales con más influencia en el sector silvoagropecuario. A nivel nacional, la superficie correspondiente a suelos erosionados (con diversos grados de erosión) es de unos 36,8 millones de hectáreas, equivalentes al $49 \%$ del territorio nacional. Los sectores con mayores problemas de erosión, por orden de importancia, son las regiones de Coquimbo con el $84 \%$ de sus suelos erosionados, Valparaíso (57\%) y O'Higgins (52\%).

La región mediterránea de Chile se extiende entre $\operatorname{los} 30^{\circ}$ y $38^{\circ}$ de latitud sur y según Costa (2007), posee alrededor de 45 endemismos. Además es la que concentra gran parte de la producción agrícola del país. En este bioclima se encuentra ubicada la ciudad de Santiago (Fig.1), cuyo principal curso fluvial es el río Maipo, que recibe como tributario la subcuenca del Puangue, la cual actualmente está presionada por el incremento de los cultivos intensivos producto de la migración de las siembras a las zonas periféricas de la ciudad (ODEPA, 2012).

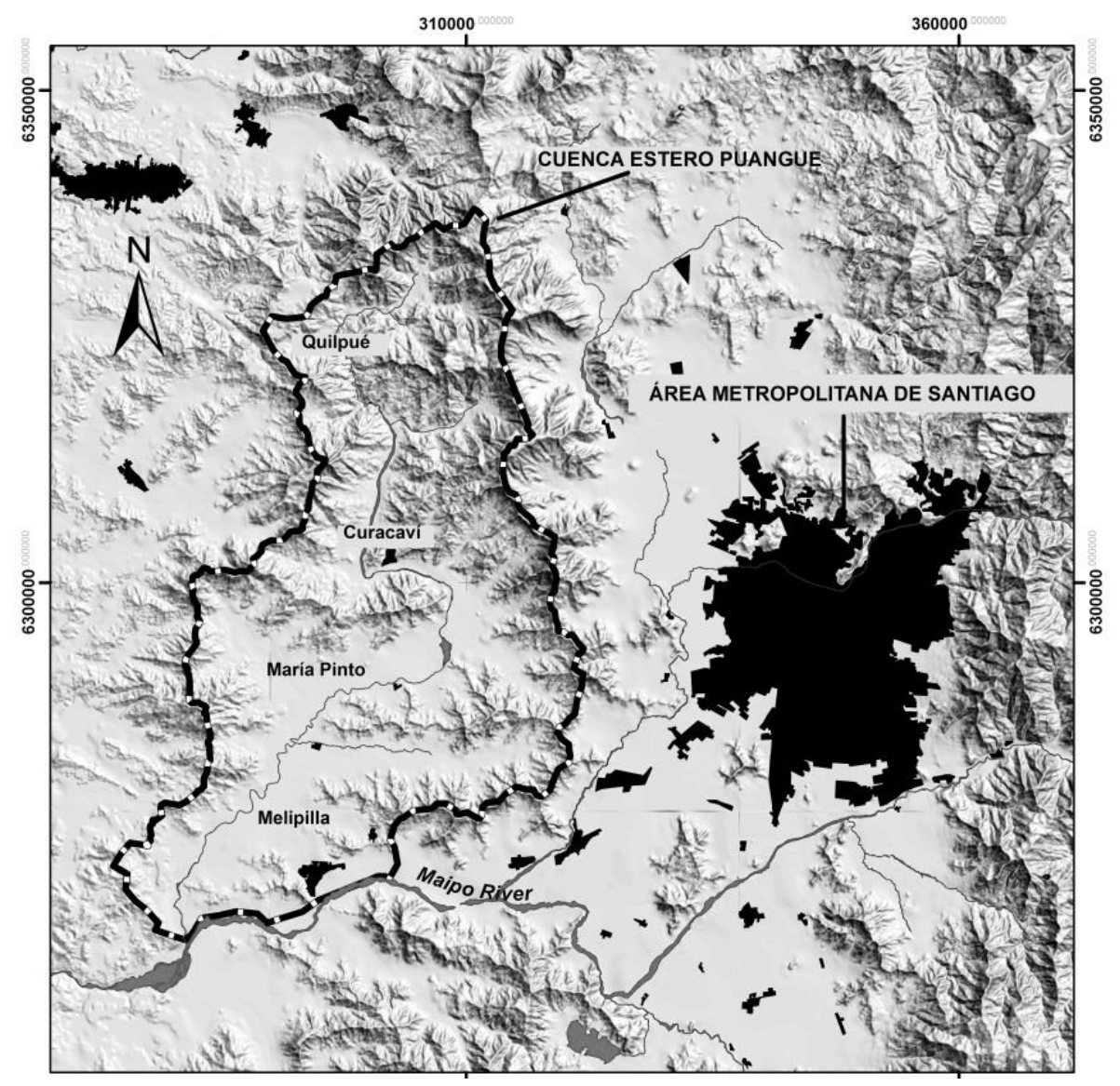

Figura 1. Ubicación del área de estudio con relación al área metropolitana de Santiago. Fuente: Elaboración del autor 
Según la CONAF (2007), todos los municipios rurales en la Región Metropolitana de Santiago de Chile poseen algún grado de desertificación, ya sea de carácter grave, moderado o leve. Este dato cobra mayor relevancia si se considera que este lugar abastece de productos agrícolas a la ciudad de Santiago, la más poblada del país. Los resultados del Censo Agropecuario (2007) señalan que del uso de suelo destinado a cultivos en la región, un 73,84\% son cultivos anuales y permanentes.

La agricultura intensiva es un tipo de producción que sin un manejo adecuado puede acentuar los procesos de desertificación, por lo que es necesario considerar que esta actividad corresponde a una agricultura industrial que requiere de mayores cantidades de agua, una fuerte mecanización y que utiliza productos agroquímicos. Esto se traduce en un aumento de la presión que la actividad agrícola ejerce sobre el medio ambiente en general y sobre el suelo en particular (Martín de Santa Olalla et al., 2001).

Mendizabal y Puigdefábregas (2003) argumentan que para la cuenca del Mediterráneo la desertificación está dada por causas endógenas (políticas agrícolas y demográficas), así como exógenas (el clima). A través del siguiente ejercicio se aplicará la metodología ESA (Kosmas et al., 1999) para identificar las dinámicas espaciales y temporales de la sensibilidad a la desertificación entre el período 1986 - 2016 en la cuenca mediterránea agrícola del Puangue en Chile, en Latinoamérica.

\section{Área de estudio}

La cuenca del Puangue se localiza en la cordillera de la costa de Chile central en la Región Metropolitana. Esta región ocupa una posición mediterránea definida por los cordones montañosos que rodean la cuenca central, que refuerza la acción dominante del anticiclón del Pacífico y marca las diferencias estacionales, con veranos cálidos y secos e inviernos fríos con lluvias esporádicas que mantienen la condición de semiaridez dentro de esta área transicional que se extiende entre los $32^{\circ} 55^{\prime}$ y $34^{\circ} 19^{\prime}$ de latitud sur. Esta zona actualmente posee una población aproximada de 45.000 habitantes y comprende los municipios de Curacaví, María Pinto, el norte de la comuna de Melipilla y parte del sector cordillerano de Quilpué (Fig.1).

\section{El uso de suelo agrícola en el Puangue}

La actividad productiva en la cuenca del Puangue se ha intensificado debido al modelo agroexportador instalado en los últimos años en el país y es posible constatar que a pesar del aumento de la urbanización que tiene la Región Metropolitana, en esa zona la producción agrícola se ha incrementado impactando al recurso hídrico y al suelo. Lo anterior es señalado en un estudio de la ODEPA (2012), según el cual las zonas de cultivo que han sido utilizadas para uso urbano han migrado hacia aquellas más alejadas de los centros que presionan por expansiones urbanas. Esto explicaría, por ejemplo, por qué la superficie de la vid no ha disminuido en los últimos años en la región. La Figura 2 muestra el aumento de hectáreas en la Región Metropolitana para la producción de vides, ya que para el período 2000 al 2007/2008 se extendió en 1702,8 hectáreas. Este es uno de los usos de suelo agrícola en la cuenca del Puangue. En este sentido, Abraham (2008) menciona que asistimos a acelerados procesos de cambio en los usos del suelo que, sobrepasando cualquier intento de planificación $\mathrm{y}$ ordenamiento, responden a intereses sectoriales y marginan de la toma de decisiones a los grupos más vulnerables.

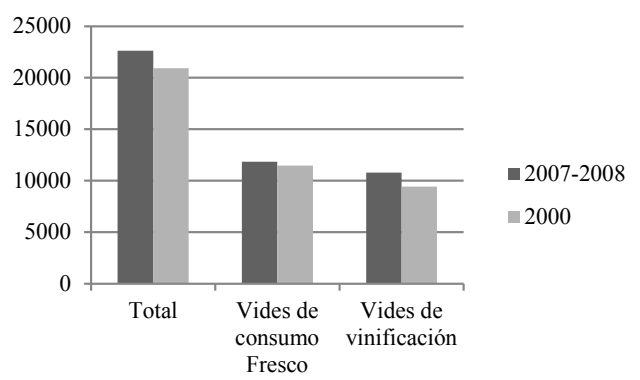

Figura 2. Superficie en hectáreas para el cultivo de la vid Región Metropolitana. Fuente: INE, 2009.

La Región Metropolitana de Santiago posee seis provincias y el área de estudio se encuentra en la provincia de Melipilla. Los datos con respecto al destino silvoagropecuario en cultivos anuales y permanentes indican que ha aumentado en 6.009 ha en el período intercensal (1997-2007), mientras que en otras provincias como la de Santiago ha disminuido en 1.858 ha la superficie de cultivos anuales y permanentes (Tabla 1).

\section{El empleo agrícola}

Los cambios en el uso de suelo a cultivos anuales y permanentes en la zona han tenido repercusiones positivas en el empleo agrícola en la provincia de Melipilla. Es necesario mencionar que esta actividad 
Tabla 1. Superficie en ha para cultivos anuales y permanentes en las provincias de la Región Metropolitana.

\begin{tabular}{lrrrrr} 
& \multicolumn{2}{c}{ Superficie (ha) } & \% cultivos anuales y permanentes \\
& $\mathbf{1 9 9 7}$ & $\mathbf{2 0 0 7}$ & $\mathbf{1 9 9 7}$ & $\mathbf{2 0 0 7}$ \\
\hline Santiago & 5.904 & 4.046 & 5 & 3 \\
Chacabuco & 13.625 & 13.056 & 6 & 7 \\
Cordillera & 5.184 & 5.455 & 1 & 1 \\
Maipo & 33.512 & 31.095 & 34 & 35 \\
\hline Melipilla & 37.381 & 43.390 & 10 & 12 \\
\hline Talagante & 18.452 & 17.500 & 37 & 39 \\
\hline Fuente: ODEPA, 2012 & & &
\end{tabular}

no requiere un mayor grado de calificación, por lo que la oferta de empleo se concentra en satisfacer la demanda de la mano de obra no calificada. La Tabla 2 muestra una variación anual positiva con un aumento de 3.935 nuevos empleos agrícolas.

Tabla 2. Empleo en la Región Metropolitana.

\begin{tabular}{|c|c|c|c|c|c|}
\hline & Personal (N) & UTE* & & & \\
\hline & 1997 & 2007 & 1997 & 2007 & $\begin{array}{r}\text { Variación empleo } \\
1997 / 2007\end{array}$ \\
\hline Santiago & 4.816 & 2.900 & 0,04 & 0,02 & $-0,4$ \\
\hline Chacabuco & 8.607 & 11.420 & 0,04 & 0,06 & 0,33 \\
\hline Cordillera & 3.669 & 3.543 & 0,01 & 0,01 & $-0,03$ \\
\hline Maipo & 20.486 & 18.159 & 0,21 & 0,21 & $-0,11$ \\
\hline Melipilla & 19.835 & 23.770 & 0,05 & 0,07 & 0,2 \\
\hline Talagante & 10.434 & 12.820 & 0,21 & 0,28 & 1,23 \\
\hline
\end{tabular}

Fuente: Odepa, 2012

* Personal de las explotaciones agropecuarias, para el caso del censo de 1997 y mano de obra en Unidad de Trabajador Equivalente (UTE), para el caso del censo de 2007. Esta última variable fue construida por Qualitas AgroConsultores para el Instituto de Desarrollo Agropecuario INDAP - con el propósito de caracterizar la pequeña agricultura familiar y otros fines.

\section{El agua en el territorio}

Tesser (2013) indica que la cantidad de agua consumida por los sectores urbanos y agrícolas en la Región Metropolitana es notoriamente diferente (Tabla 3), y el mayor consumo es generado por las actividades de la agricultura.

Tabla 3. Consumo de agua $(\mathrm{m} / \mathrm{s})$.

\begin{tabular}{rrrrrr}
\hline \multicolumn{7}{c}{ Usos del agua $\left(\mathrm{m}^{3} / \mathrm{s}\right)$} \\
Año & Energía & Agrícola & Doméstico & Industrial & Minero \\
\hline 2005 & 129 & 82,4 & 18,5 & 10,4 & 0,5 \\
2030 & 129 & 80,5 & 24,8 & 27,6 & 2 \\
\hline
\end{tabular}

A pesar de que se ha invertido en innovación tecnológica para riego con el empleo de nuevas formas de manejo del agua, la actividad agrícola continúa siendo la principal consumidora en la región. Como muestra la Tabla 4, el riego gravitacional que conlleva grandes pérdidas de agua por evaporación ha disminuido y ha aumentado el uso de microrriego, que es más eficiente y casi se cuatriplica para el período 1997-2007.

En el año 2008 la Dirección General de Aguas, mediante el Informe Técnico $\mathrm{N}^{\circ} 28$ (DGA, 2008), declaró los subsectores hidrológicos Puangue Alto y Medio como área de restricción y recomienda "no avanzar en la explotación previsible de los derechos de aprovechamiento más allá del caudal equivalente a la explotación neta sustentable en cada sector del acuífero". Lo anterior se hace con base en estudios que demuestran que existe riesgo de grave disminución de un determinado acuífero. Corresponde a una medida de carácter preventivo y no necesariamente a un deterioro real en la actualidad.

\section{Metodología}

El concepto de sensibilidad ambiental comenzó a utilizarse hace unos 30 años en los países industrializados y se ha estimulado recientemente como una forma de hacer frente a la creciente conciencia sobre la desertificación, la degradación de los suelos y sus consecuencias (Rubio, 1998; Basso et al,. 2000 y Contador, 2009).

La sensibilidad ambiental puede definirse como la respuesta del medio ambiente o parte de ella a un cambio en uno o más factores externos. Las relaciones entre la causa del cambio y el efecto son a menudo complejas, porque los componentes ambientales separados responden directamente a los cambios, pero con diferentes sensibilidades, mientras que, debido a las interrelaciones entre los componentes, también se ven afectados indirectamente. La degradación se produce cuando la respuesta se considera perjudicial para "la salud" del medio ambiente (Basso et al., 2000).

La metodología ESA -por las siglas en inglés Environmentally Sensitive Area index- fue desarrollada para el proyecto europeo occidental Mediterranean Desertification and Land Use 
Tabla 4. Superficie regada según tipo de riego.

\begin{tabular}{llrrrr}
\hline $\begin{array}{l}\text { Censo } \\
\text { Agropecuario }\end{array}$ & Nivel & \multicolumn{1}{l}{$\begin{array}{l}\text { Total superficie } \\
\text { regada }\end{array}$} & Gravitacional & \multicolumn{1}{l}{$\begin{array}{l}\text { Mecánico } \\
\text { mayor }\end{array}$} & Micro riego \\
\hline $1997 / 1998$ & Chile & $1.058 .355,9$ & $960.840,0$ & $30.522,5$ & $62.153,3$ \\
& $\begin{array}{l}\text { Región } \\
\text { Metropolitana }\end{array}$ & $144.844,5$ & $127.613,7$ & $5.983,9$ & $11.246,9$ \\
\hline & Chile & $1.093 .812,4$ & $789.839,9$ & $56.498,3$ & $247.474,2$ \\
& $\begin{array}{l}\text { Región } \\
\text { Metropolitana }\end{array}$ & $136.732,3$ & $90.890,1$ & $3.799,8$ & $42.042,4$ \\
\hline
\end{tabular}

Fuente: ODEPA, 2012

(MEDALUS) por Kosmas et al., (1999) y consiste en un set de indicadores básicos (Tabla 5) para la identificación de las áreas ambientalmente sensibles a la desertificación. Estos sectores son definidos como pequeñas áreas que realizan funciones ambientalmente críticas en un paisaje más amplio. Las áreas identificadas con esta metodología desempeñan funciones ecológicas vitales que se consideran esenciales para mantener la integridad de los ecosistemas y la resiliencia.

Esta metodología ha sido validada tanto en escalas regionales como en locales, especialmente en la cuenca del Mediterráneo europeo (Portugal, España, Italia y Grecia) y bajo diferentes condiciones ambientales (Salvati y Bajocco, 2011). Ejemplo de su aplicación han sido los trabajos realizados por Basso et al. (2000), Farajzadeh y Nik Egbal (2007), Contador, et al. (2009), Hadeel, et al. (2010) y Salvati y Bajocco (2011).

\section{Índice de sensibilidad ambiental a la desertificación}

El índice está compuesto por cuatro subíndices:

Subíndice calidad de suelo (Ss). En las tierras secas, la desertificación puede ser irreversible cuando la profundidad del suelo no es capaz de mantener un mínimo de cobertura vegetal. Hay casos en que procede la desertificación en suelos profundos, cuando su balance de agua es incapaz de satisfacer las necesidades de las plantas. Los indicadores para medir cambios en la calidad del suelo son:

$$
\begin{aligned}
& \text { Ss }=(\text { textura } * \text { pendientes } * \text { pedregosidad } * \\
& \text { profundidad de suelo*drenaje }) 1 / 5
\end{aligned}
$$

Subíndice calidad de clima (Sc). La desigual distribución anual e interanual de las precipitaciones y los fenómenos extremos y de temporadas de lluvias en las zonas semi-áridas y áridas en los climas mediterráneos son las principales características climáticas que contribuyen a la degradación de la tierra. El suelo en estas zonas climáticas es inestable y los procesos de desertificación se activan solo si los demás componentes de la tierra sobrepasan los umbrales específicos. El cambio climático mundial espera ampliar la actual geografía de las zonas sensibles en las zonas mediterráneas.

$$
S c=(\text { precipitaciones } * \text { aridez } * \text { orientación }) \text { 1/3 }
$$

Subíndice de calidad de la vegetación (Sv). $\mathrm{La}$ cubierta vegetal es crucial para la generación de escorrentía y puede ser fácilmente modificada a lo largo de las zonas con clima mediterráneo y en las zonas montañosas, según las condiciones climáticas y la época del año. En las áreas con precipitación anual inferior a $300 \mathrm{~mm}$ y con una alta tasa de evapotranspiración, el agua disponible en el suelo para las plantas se reduce drásticamente causando un alto estrés hídrico.

\section{$S v=($ riesgo de incendios*protección a la erosión*resistencia a la sequía*cobertura vegetal) $1 / 4$}

Subíndice de calidad de la gestión (Sg). Un área del territorio se caracteriza por un uso particular. Este uso se asocia con una determinada actividad que es dictada por los cambios y bajo la influencia del medio ambiente y los factores tecnológicos y políticos.

$$
\mathrm{Sg}=(\text { Intensidad en el uso de suelo } * \text { Políticas })^{1 / 2}
$$

Cada indicador tiene diferentes ponderadores según diferentes parámetros, asignándoles de acuerdo a sus características un peso (Tabla 5). Una vez que a cada unidad se le asigna un peso, a través de la metodología de álgebra de mapas se aplica la fórmula:

$$
\text { Índice de sensibilidad ambiental }=\left(\mathrm{Sc}^{*} \mathrm{Sc} * \mathrm{Sg} * \mathrm{~Sv}\right)^{1 / 4}
$$

Una vez obtenidos los resultados de la aplicación algebraica, se identifican áreas de acuerdo con los parámetros que muestra la Tabla 6. 


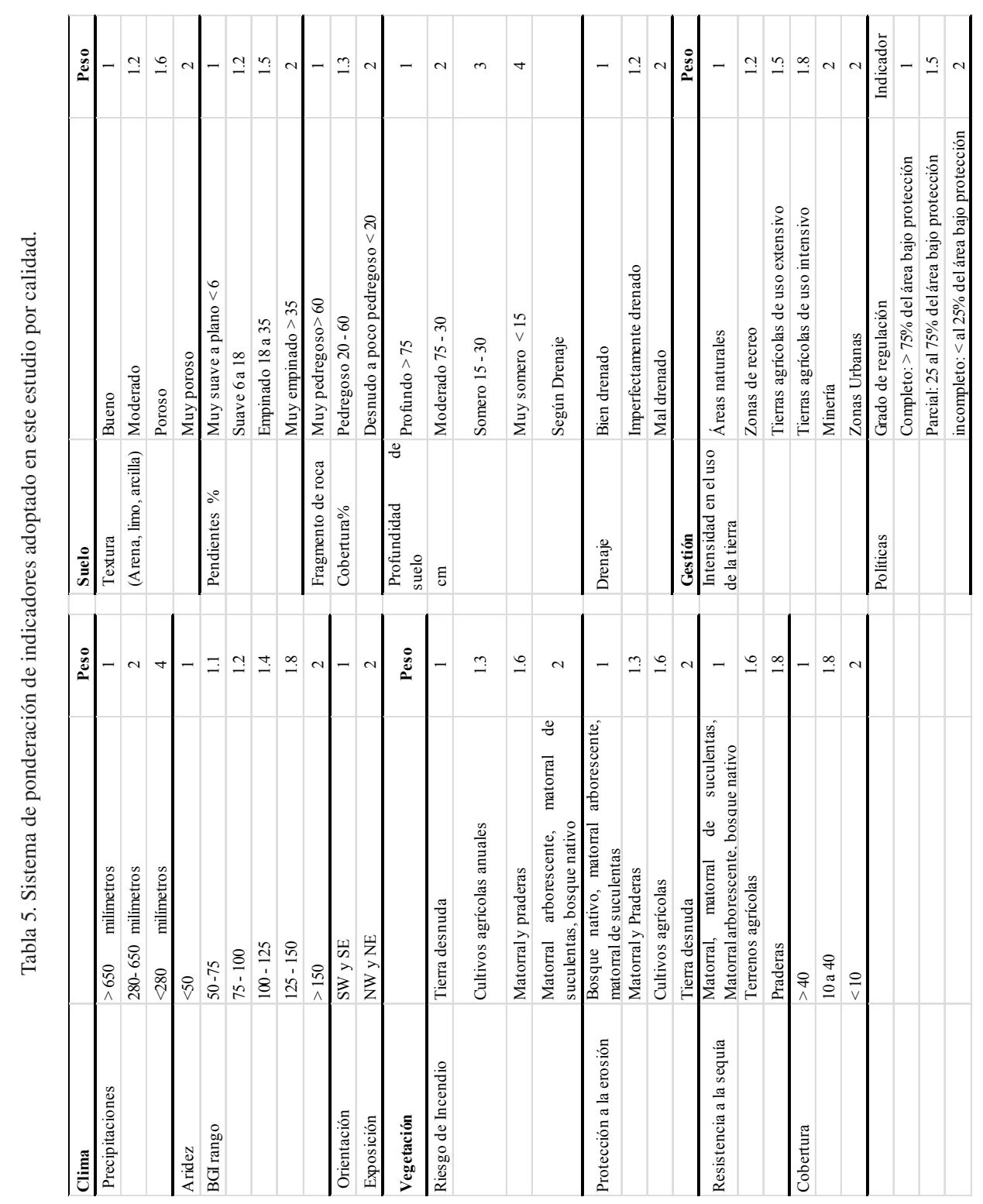


Tabla 6. Clasificación del área de estudio en términos de la sensibilidad a la desertificación.

\begin{tabular}{rlcc} 
& \multicolumn{1}{c}{ Tipo } & Subtipo & Rango ESAS \\
\cline { 3 - 4 } Críticas & Crítica & $\mathrm{C} 3$ & $>1,53$ \\
& Crítica & $\mathrm{C} 2$ & $1,42-1,53$ \\
Crítica & $\mathrm{C} 1$ & $1,38-1,41$ \\
Prágiles & Frágil & $\mathrm{F} 1$ & $1,23-1,37$ \\
No afencial & Potencial & $\mathrm{P}$ & $1,17-1,22$ \\
No afectada & $\mathrm{N}$ & $<1,17$
\end{tabular}

Otro aspecto a considerar para la gestión de la cuenca es que su superficie depende administrativamente de cuatro municipios: Quilpué, Curacaví, María Pinto y Melipilla. Por ello fue necesario separar las superficies en sus distintos grados de sensibilidad según su división política administrativa para cuestiones de análisis.

\section{Resultados y discusión}

\section{Evolución de la sensibilidad ambiental a la desertificación en la cuenca del Puangue}

Las ESA se calcularon sobre la base de la técnica de superposición de los diferentes indicadores en relación con su calidad, que además permitió la identificación de los vínculos entre estos y sus patrones espaciales.

La aplicación del índice arrojó un aumento en el número de hectáreas que presentan algún grado de sensibilidad a la desertificación, ya sea en las categorías potenciales, frágiles o críticas en el período 1986-2016. La ubicación espacial de estas áreas está asociada a los sectores adyacentes al estero Puangue, donde los cultivos agrícolas se han extendido hacia las laderas de los cordones montañosos. En este sentido, se puede mencionar que el uso de suelo destinado a la agricultura se relaciona con la configuración orográfica de la cuenca, ya que en el año 1986 el uso agrícola estaba concentrado en las terrazas fluviales, mientras que en los últimos años los cultivos han reemplazado a la vegetación nativa dando paso a una agricultura altamente intensiva y tecnificada en sectores de ladera. El aumento de la sensibilidad ambiental a la desertificación en el área es reflejo de estos cambios espaciales, por lo que se prevé que estos procesos pueden acentuarse, debido principalmente a la falta de planificación territorial y fiscalización del área rural. Por ejemplo, entre 1986-2016 el número de hectáreas que pasaron a críticas fue de 7.393, lo que equivale a un aumento del $53,4 \%$. Este incremento en el número de hectáreas que presentan algún grado de sensibilidad se ha mantenido sostenido en el tiempo (Fig. 3)

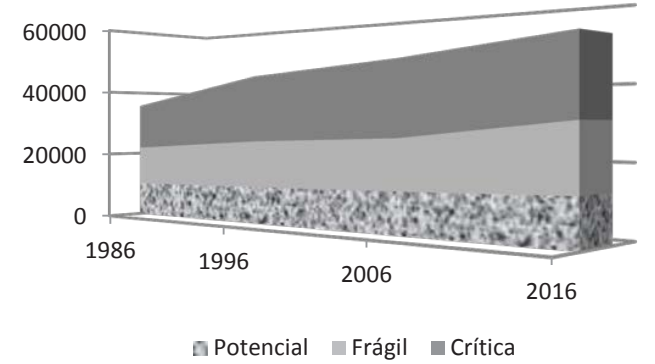

Figura 3. Evolución del número de hectáreas según categorías: potenciales, frágiles y críticas por año. Fuente: Elaboración propia

La Figura 4 muestra que en todos los años analizados se aprecia el aumento de las áreas afectadas en las diferentes categorías de sensibilidad. De igual manera destacan las superficies críticas que han aumentado especialmente en el año 2016. Ello permite situar a esta cuenca como un área que ha experimentado en los últimos 30 años un sostenido incremento de la sensibilidad, tal como ha ocurrido en otros lugares del mundo monitoreados con la misma metodología.

Desde el punto de vista político administrativo, la cuenca del Puangue presenta las mayores superficies con sensibilidad crítica en los municipios de María Pinto y en el área de estudio de la localidad de Mallarauco en Melipilla, por lo que es en estas zonas donde hay que poner mayor atención a las medidas de manejo del suelo (Figura 4).

\section{Conclusiones}

El proceso paulatino de industrialización de la agricultura en la zona central de Chile ha traído externalidades que han sido escasamente monitoreadas. A ello se le suma la presión por el uso de suelo desde el sector inmobiliario en la periferia de la metrópolis de Santiago y por ende la migración de los cultivos a la zona semiárida costera de la Región Metropolitana. La cuenca del Puangue no ha estado ajena a los procesos anteriores, y esto se ha visto reflejado en el aumento de la superficie cultivada y el de las áreas en condiciones "críticas" al aplicar el modelo de sensibilidad ambiental a la desertificación.

De acuerdo con lo anterior, la desertificación es un proceso silencioso que está afectando al mediterráneo chileno. Una prueba de ello es el aumento de la 

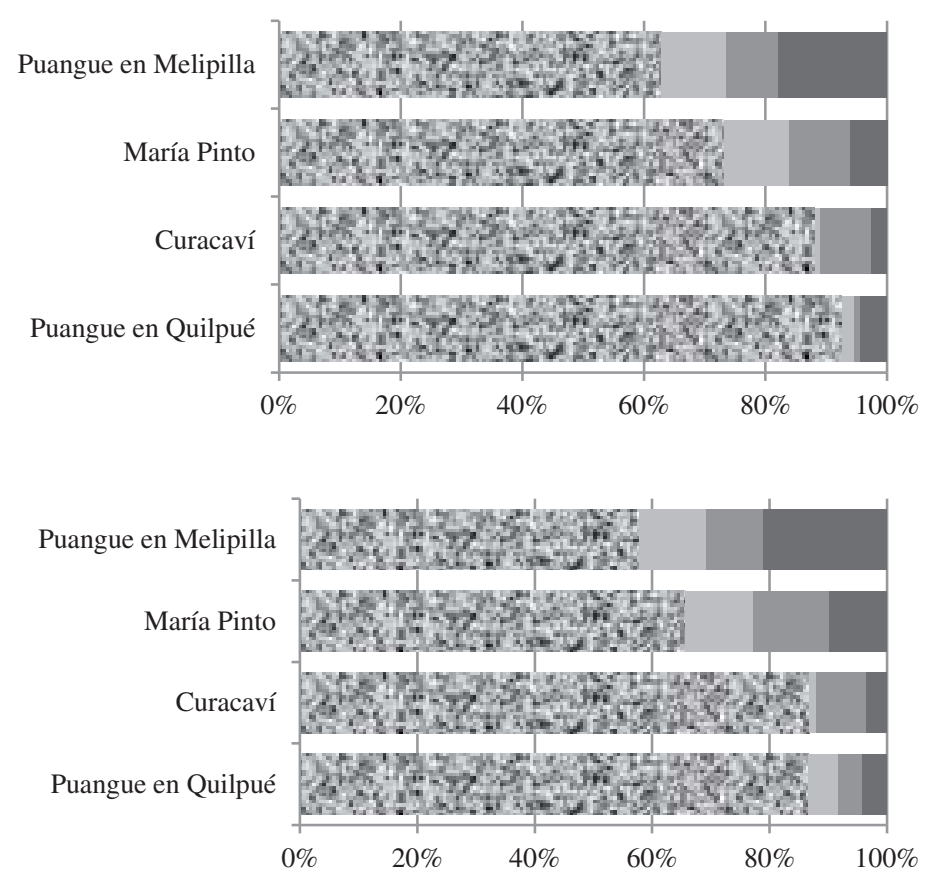

1996
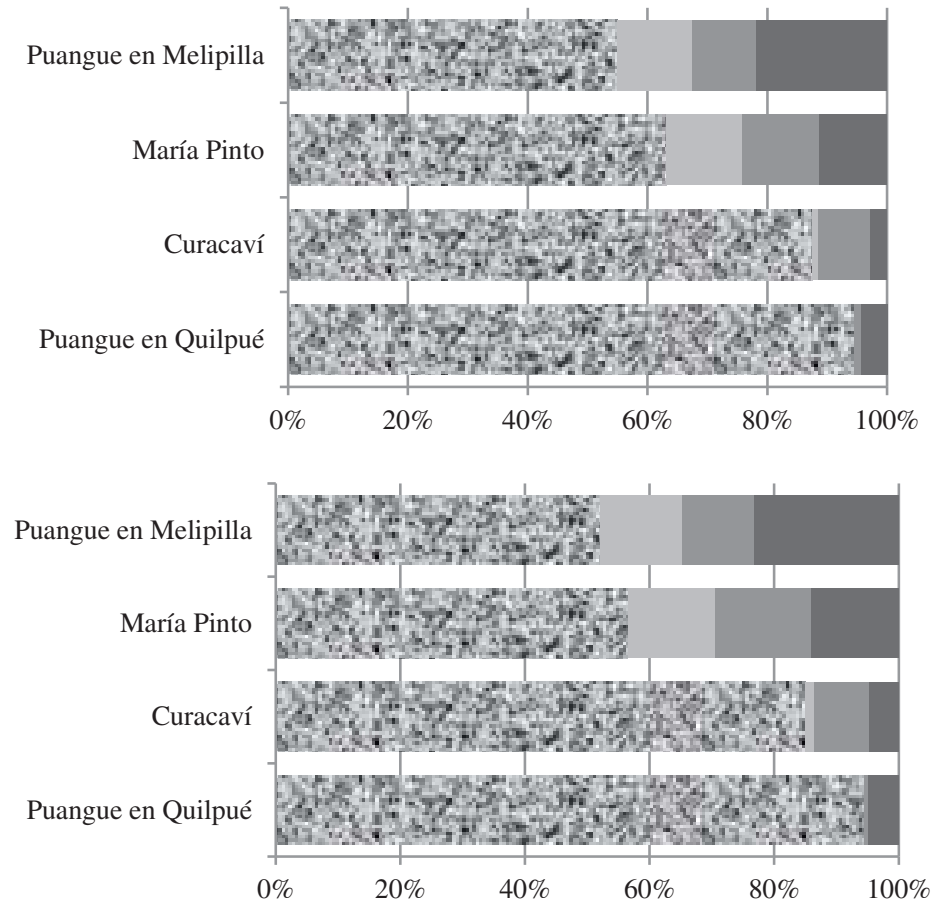

$\square$ No afectadas $\square$ Potenciales $\square$ Frágiles $\square$ Críticas

Figura 4. Evolución de la sensibilidad a la desertificación de la cuenca del Puangue según división política administrativa 
sensibilidad ambiental. Los cambios en la cuenca se asocian también a transformaciones espaciales de los usos de la tierra. Es por ello que una eficiente gestión y monitoreo de los instrumentos de ordenamiento territorial y la fiscalización son primordiales para disminuir los niveles de sensibilidad, especialmente en la superficie de las comunas cuyos resultados fueron críticos como María Pinto y el sector de Mallarauco en Melipilla.

\section{Agradecimientos}

La autora quiere agradecer al Doctorado en Medio Ambiente y Desarrollo de la Universidad Autónoma de Baja California en México, a CONACYT (México), a CONICYT (Becas Chile), así como a las actuales autoridades locales de la comuna de María Pinto, que han apoyado esta investigación y están velando por la sustentabilidad de la cuenca del Puangue.

\section{Literatura citada}

Abraham, E.

2008. Tierras secas, desertificación y recursos hídricos. Ecosistemas, 17 (1): 1-4.

Basso, F.; Bovea E.; Dumonteta S.; Ferraraa A.; Pisantea M.; Quarantaa G.; Tabernerb M.

2000. Evaluating environmental sensitivity at the basin scale through the use of geographic information systems and remotely sensed data: an example covering the Agri basin (Southern Italy). Catena, 40 (1): 19-35.

INE.

2007. Censo Agropecuario y Forestal. Instituto Nacional de Estadísticas. Disponible en: http://www.ine.cl/estadisticas/ censos/censo-agropecuario-y-forestal-2007. Consultado: 15/sep/2017.

CONAF,

1999. Mapa preliminar de la desertificación en Chile por comunas. Soto G. (Ed.), Coordinador Nacional Programa Acción Nacional contra la Desertificación. La Serena, Chile, 88 p.

Congreso Nacional de Chile.

2012. La desertificación en Chile. Unidad de Diagnóstico Parlamentario. Departamento de Evaluación de la Ley. Valparaíso, Chile. 20 p.

Contador, J.; Schnabel S.; Gómez Gutiérrez A.; Pulido M.

2009. Mapping sensitivity to land degradation in Extremadura SW Spain. Land Degradation \& Development, 20 (2): 129-144.

Costa, M.

2007. Diversidad paisajística y vegetacional en el Mediterráneo. Cuadernos de sostenibilidad y Patrimonio Natural, 11: 129-137.

DGA.

2008. Informe técnico N 128: Declaración área de restricción subsectores hidrogeológicos de aprovechamiento común de Puangue Alto, Puangue Medio, Cholqui, Popeta, Melipilla y La Higuera del acuífero de Puangue Melipilla. Ministerio de Obras Públicas, Dirección General de Aguas, Departamentos de Administración de Recursos Hídricos. Santiago, Chile. 39 p.

Farajzadeh M.; Nik Egbal M.

2007. Evaluation of MEDALUS model for desertification hazard zonation using GIS; Study area: Iyzad Khast Plain, Iran. Pakistan Journal of Biological Science, 10 (16): 2622-2630.
Gardi, C.; Angelini, M.; Barceló, S.; Comerma, J.; Cruz G.; Encina, A.; Jones, A.; Krasilnikov, P.; Mendonça, M.; Montanarella, L.; Muñiz, O.; Schad, P.; Vara, M.; Vargas, R.

2014. Atlas de suelos de América Latina y el Caribe, Comisión Europea - Oficina de Publicaciones de la Unión Europea. 176 p.

Hadeel, S.; Jabbar M.; Chen X.

2010. Application of remote sensing and GIS in the study of environmental sensitivity to desertification: a case study in Basrah Province, southern part of Iraq. Applied Geomatics, 2 (3): 101-112.

INE.

2009. Catastro Vitícola. Informe Anual. Instituto Nacional de Estadísticas y Servicio Agrícola y Ganadero. Gobierno de Chile. Disponible en: http://www.ine.cl/estadisticas/ economicas/estad\%C3\%ADsticas-agropecuarias. Consultado: 23/sep/2017.

IPCC .

2014. Cambio climático 2014. Impactos, adaptación y vulnerabilidad. Contribución del Grupo de trabajo II al Quinto Informe de Evaluación del Grupo Intergubernamental de Expertos sobre el Cambio Climático. Suiza. 32 p.

Kosmas, C.; Krikby M.; Geeson N.

1999. Methodology for Mapping Environmentally Sensitive Areas (ESAs) to Desertification. En: Kosmas, C.; Kirkby, M.; Geeson, N. (Eds.). The Medalus Project Mediterranean Desertification and Land Use. Manual on Key Indicators of Desertification and Mapping Environmentally Sensitive Areas to Desertification. EU: Brussels, Belgium. pp. 31-47. Martín de Santa Olalla, F.

2001. Agricultura y desertificación. Mundi-Prensa. Madrid, España. 341 p.

Mendizabal, T.; Puigdefábregas, J.

2003. Population and Land-use Changes: Impact on Desertification in Southern Europe and in the Maghreb. En: Brauch, Liotta, Marquina, Rogers, Selim (Eds.): Security and Environment in the Mediterranean. Springer. BerlinHeidelberg, Germany. pp. 687-701. ODEPA

2012. Estudio de impacto de la expansión urbana sobre el sector agrícola en la Región Metropolitana de Santiago. Estudio Comparativo de las transformaciones del sector agrícola relacionadas con el avance de la urbanización. Regiones: Metropolitana, VI, VII y VIII. Oficina de Estudios y Políticas Agrarias. Ministerio de Agricultura. Santiago, Chile. 54 p. 
Pizarro, R.; Morales, C.; Vega, L.; Valdés, R.; Olivares, C.; Balocchi, F. 2010. Evaluación de la Erosión Hídrica Superficial en Zonas Áridas y Semiáridas de Chile Central. Aqua-LAC, 2 (2) 1- 11. Rubio, J. L., y Bochet, E.

1998. Desertification indicators as diagnosis criteria for desertification risk assessment in Europe. Journal of Arid Environments, 39 (2), 113-120.

Salvati, L.; Bajocco, S.

2011. Land sensitivity to desertification across Italy: Past, present, and future. Applied Geography, 31 (1): 223-231.

Tesser C.

2013. El agua y los territorios hídricos en la Región Metropolitana de Santiago de Chile. Casos de estudio: Tiltil, Valle de Mallarauco y San Pedro de Melipilla. Estudios Geográficos, 74 (274): 255-285.

\section{UNCCD.}

2011. Desertificación: Una síntesis visual. UNCCD Publications. $50 \mathrm{p}$.

UNEP.

1992. World Atlas of Desertification (United Nations Environment Programme). En Middleton N. y Thomas D. S. G. (Eds). Edward Arnold. Londres, UK. 69 p.

Universidad de Chile.

1997. Diagnóstico de la Desertificación en Chile. Santiago, Chile. 399 p.

Verbist, K.; Santibáñez, F.; Gabriels, D.; Soto G.

2010. Atlas de Zonas Áridas de América Latina y el Caribe. CAZALAC. Documentos Técnicos del PHI-LAC. Montevideo, Uruguay. 48 p. 